\title{
A compact time-resolved system for near infrared spectroscopy based on wavelength space multiplexing
}

\author{
Rebecca Re, ${ }^{1, a)}$ Davide Contini, ${ }^{1}$ Matteo Caffini, ${ }^{1}$ Rinaldo Cubeddu, ${ }^{1,2,3}$ Lorenzo Spinelli, ${ }^{2}$ \\ and Alessandro Torricelli ${ }^{1,3}$ \\ ${ }^{1}$ Dipartimento di Fisica, Politecnico di Milano, Piazza Leonardo da Vinci 32, I-20133 Milan, Italy \\ ${ }^{2}$ IFN-CNR, Istituto di Fotonica e Nanotecnologie, Piazza Leonardo da Vinci 32, I-20133 Milan, Italy \\ ${ }^{3}$ Research Unit IIT, Politecnico di Milano, Piazza Leonardo da Vinci 32, I-20133 Milan, Italy
}

(Received 13 July 2010; accepted 23 August 2010; published online 2 November 2010)

\begin{abstract}
We designed and developed a compact dual-wavelength and dual-channel time-resolved system for near-infrared spectroscopy studies of muscle and brain. The system employs pulsed diode lasers as sources, compact photomultipliers, and time-correlated single photon counting boards for detection. To exploit the full temporal and dynamic range of the acquisition technique, we implemented an approach based on wavelength space multiplexing: laser pulses at the two wavelengths are alternatively injected into the two channels by means of an optical $2 \times 2$ switch. In each detection line (i.e., in each temporal window), the distribution of photon time-of-flights at one wavelength is acquired. The proposed approach increases the signal-to-noise ratio and avoids wavelength cross-talk with respect to the typical approach based on time multiplexing. The instrument was characterized on tissue phantoms to assess its properties in terms of linearity, stability, noise, and reproducibility. Finally, it was successfully tested in preliminary in vivo measurements on muscle during standard cuff occlusion and on the brain during a motor cortex response due to hand movements. (C) 2010 American Institute of Physics. [doi:10.1063/1.3495957]
\end{abstract}

\section{INTRODUCTION}

Noninvasively monitoring of blood and tissue oxygenation is one of the research field, where near-infrared spectroscopy (NIRS) can be applied. ${ }^{1-3}$ This technique was utilized both to experimentally and clinically investigate cerebral oxygenation and hemodynamic response to a wide range of stimuli in the human brain ${ }^{4,5}$ and to study muscle oxidative metabolism in healthy and pathological subjects. ${ }^{6}$

Different typologies of NIRS measurements are nowadays available: continuous wave, frequency, and time domain. ${ }^{7-13}$ The time-domain approach to NIRS potentially allows to discriminate between absorption and scattering contributions and to calculate absolute values for the hemodynamic parameters, ${ }^{14}$ compared to the cw modality. Moreover, it has the advantage of improving the depth sensitivity by exploiting the temporal information, ${ }^{15-18}$ compared to frequency domain measurements.

While several multichannel systems for time-resolved NIRS have been reported in literature and successfully used for imaging studies, ${ }^{19-22}$ in particular on the brain, there are clinical applications where no imaging capabilities are required and an instrument with a limited number of channels can be effectively used for monitoring pathophysiological changes. For example, in motor rehabilitation of stroke patients, we have employed time-resolved NIRS to quantify the progress of training protocols by measuring the muscle hemodynamics using a limited number of channels. ${ }^{23}$ Moreover, this reduces the size and the cost of the instrument.

\footnotetext{
${ }^{\text {a)} E l e c t r o n i c ~ m a i l: ~ r e b e c c a . r e @ ~ m a i l . p o l i m i . i t . ~}$
}

In this work, we present the development of a compact dual-wavelength and dual-channel time-resolved NIRS system, where a new modality to inject light into the tissue is introduced: the wavelength space multiplexing (SM). The aim of our study was to improve the performances of compact time-resolved systems in terms of signal-to-noise ratio and temporal dynamic range of the measurements. Our novel approach allows, indeed, to exploit the full temporal dynamic range of the acquisition boards, thus increasing the signal-to-noise ratio and avoiding wavelengths cross-talk with respect to the typical approaches based on wavelength time multiplexing (TM).

We will describe the SM approach in Sec. II A, and the system setup in Sec. II B. The characterization of the instrument on tissue phantoms and preliminary in vivo measurements during a standard cuff occlusion and a motor cortex response are respectively described in Secs. III A and III B. Finally, in Sec. IV, a comparison between the SM and TM approaches is presented.

\section{SYSTEM SETUP}

\section{A. The wavelength space multiplexing approach}

Typically, systems based on time-resolved NIRS use a TM approach to inject light at two different wavelengths into the tissue. As shown in Fig. 1(a), the optical pulses at different wavelengths are delayed, one with respect to the other, by means of an optical fiber delayer line and mixed by means of an optical coupler ${ }^{21}$ or a fiber optical splitter, ${ }^{20-22}$ which halves the intensity of the pulses. In this way, it is possible to time-multiplex the wavelengths which are both acquired in the same temporal window. The information carried by the 


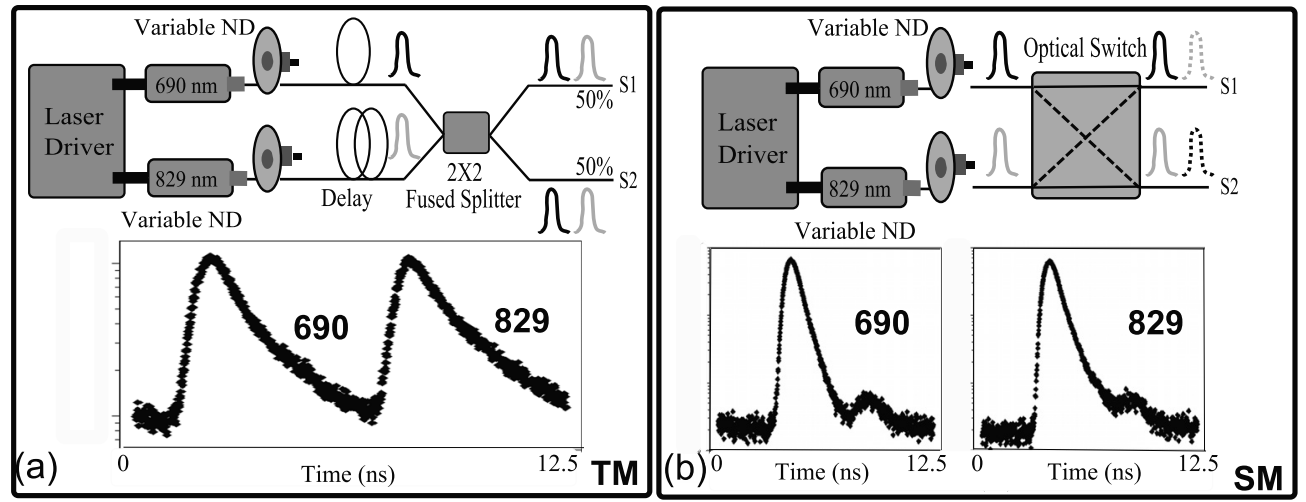

FIG. 1. Comparison between the two different injection approaches and how the acquired curves appear in a temporal window. (a) TM, (b) SM.

curves is acquired simultaneously in time, but problems of cross-talk between pulses might hamper the overall performances of the system. Cross-talk could be due to the superposition of the distributions of photon time-of-flight (DTOFs). Nonlinearity in the time-to-amplitude converter might also reduce the available time-interval in the timecorrelated single photon counting (TCSPC) apparatus. In case of large source detector distance and/or low absorption coefficient, the DTOFs are rather broad and this could result in a partial overlapping of the DTOFs itself. Another source of cross-talk might arise from significant changes in the counts at one wavelength, in particular when the count rate is high and when in the system, a routing electronics is used to manage signals coming from different detectors. In these cases, it is necessary to keep the count rate low with an overall reduction of the performances.

To avoid wavelength cross-talk and to increase signal-tonoise ratio, we have introduced a new approach to inject and collect light into the tissue based on wavelength SM [Fig. 1(b)]. The two wavelengths are injected alternatively into the two channels by means of an optical $2 \times 2$ switch. In this way, we acquire in each detection line and in each temporal window only one DTOF at one wavelength at a time. Using this new approach is possible to exploit the full temporal and dynamic range of the acquisition TCSPC boards. Due to the fact that the wavelengths are not simultaneously measured at the same point, the switching rate must be sufficiently high to follow the hemodynamic changes in the tissue under examination (e.g., for the brain, $5 \mathrm{~Hz}$ sampling rate can be enough).

\section{B. System description}

In Fig. 2, a complete scheme of the instrument setup is reported. As light sources, a couple of pulsed diode lasers, working in gain switching modality, operating at 690 and $829 \mathrm{~nm}$ with $80 \mathrm{MHz}$ repetition rate (PDL, Picoquant $\mathrm{GmbH}$, Germany) are used. After the laser heads, light is injected into multimode graded index fibers $(50 / 125 \mu \mathrm{m})$ by means of a custom-made coupler. It combines a couple of neutral density attenuators (NT43-770, Edmund Optics $\mathrm{GmbH}$, Germany), with a total variable attenuation in the range of $0-80 \mathrm{~dB}$, and a standard fiber optics coupler. Variable attenuators are needed during measurements on biological tissues to equalize the signal of the two wavelengths or during the acquisition of the instrument response function (IRF) to avoid damages of the detectors. The attenuators are automatically controlled by stepper motors (440-436, RS Components, Italy). After the attenuation stage, light passes through an optical $2 \times 2$ switch (LEONI Fiber Optics GmbH, Germany, maximum switching of rate $5 \mathrm{~ms}$ ), which allows the wavelength SM.

Photons reemitted by the sample are collected by two custom-made glass fiber optic bundles (Loptek Glasfasertechnick GmbH, Germany) with $3 \mathrm{~mm}$ diameter and 0.49 numerical aperture. After the bundles, the signal is equalized by variable neutral density attenuators (like in the acquisition section). Then, the signal is coupled to the active area of a photomultiplier tube (PMT) (PMC-100, Becker\&Hickl, Germany) by means of a proper optical lens system. This is composed of two aspherical lenses with a focal lengths of 18.75 and $25 \mathrm{~mm}$, respectively (NT-47-729/730, Edmund Optics Gmbh, Germany), arranged inside a C-mount tube. This configuration allows to reach a coupling efficiency of $96 \%$, keeping the dimensions of the entire optical system around $52 \mathrm{~mm}$ (from bundle to the active area of the PMT). The PMTs are cooled by an internal Peltier system driven by external home-made circuits. After the PMTs, the electrical signals are amplified (HAFC-26, Becker\&Hickl, Germany).

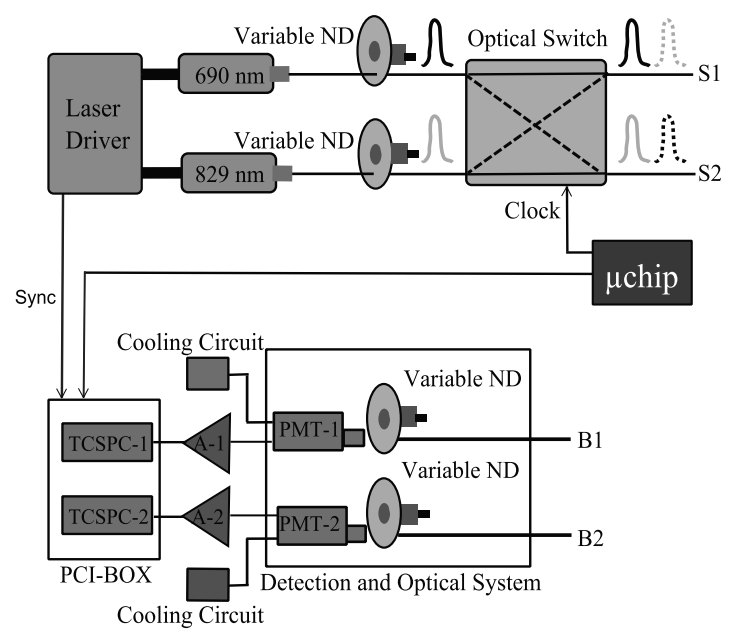

FIG. 2. Overview of the entire system. ND: neutral density attenuator; S: injection fibers; B: collection bundle; PMT: photomultiplier tube; A: amplifier; TCSPC: time correlated single photon counting board; $\mu$ chip: microcontroller unit; and Sync: synchronization signal. 


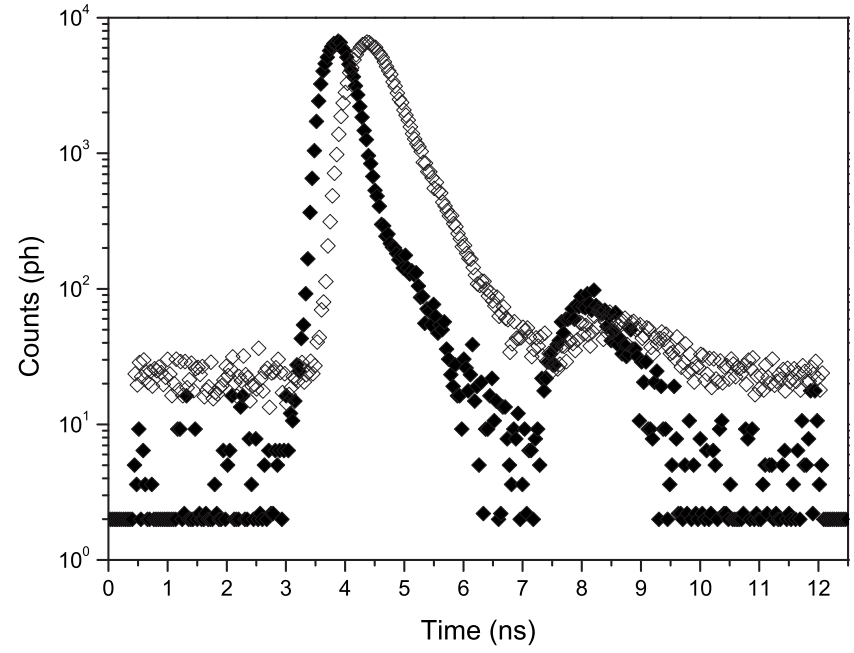

FIG. 3. Example of the instrument response function (black points) and of a time-resolved reflectance curve (open symbols) at $690 \mathrm{~nm}$.

The acquisition of time-resolved reflectance curves is accomplished by two parallel and identical boards (SPC130, Becker\&Hickl, Germany) for TCSPC. ${ }^{24}$ They are placed in a PCI-box (PCI-Cardbox 2F, IBP Instruments Gmbh, Germany) interfaced with a laptop and have a useful count rate of $4 \mathrm{MHz}$ each. The maximum count rate and the commutation time of the optical switch $(5 \mathrm{~ms})$ impose a minimum in the acquisition time of about $10 \mathrm{~ms}$ to have a reliable (about $210^{4}$ counts per curve) measurement.

All the system is controlled by a home-made software, written in C-language in the LabWindows/CVI environment (National Instruments, Austin, TX), interfaced to a microcontroller unit (dsPIC30F2010, Microchip Technology, Inc., Chandler, AZ). The microcontroller unit receives, through a serial communication, the settings parameters from the laptop and provides a trigger signal to synchronize the optical switch and the data acquisition of the TCSPC boards. The system is also provided by suitable probes (made of Velcro stripes and custom-made plastic holders) for positioning of the injection fibers and of the collection bundles on the tissue.

In Fig. 3, typical instrument response function (IRF, black points) and DTOF curve (open symbols) at $690 \mathrm{~nm}$ are shown. The full width at half maximum of the IRF is approximately 570 ps. Similar characteristics were observed for the other wavelength.

\section{SYSTEM CHARACTERIZATION}

\section{A. Medphot protocol}

A standard characterization protocol was employed to evaluate the system's performances in terms of measurement results and to make it comparable with other photon migration instruments. The applied protocol, developed within the framework of the European thematic Network "Medphot," 25 schedules to measure a set of 32 solid phantoms based on epoxy resin with $\mathrm{TiO}_{2}$ powder as the scatterer and black toner as the absorber. Each phantom has a label composed of a letter (A-D) and a number (1-8) representing, respectively, the nominal reduced scattering (5-20 and $5 \mathrm{~cm}^{-1}$ steps) and the nominal absorption (0-0.49 and $0.07 \mathrm{~cm}^{-1}$ steps) coefficients at $660 \mathrm{~nm}$. A simultaneous estimate of reduced scattering coefficient and absorption coefficient for each sourcedetector couple was achieved by best fitting of DTOFs with a standard model of diffusion theory. ${ }^{26}$

\section{Linearity of $\mu_{a}$ and $\mu_{s}^{\prime}$}

Reflectance measurements on all the phantoms and for all the injection and detection lines were performed with an interfiber distance of $2 \mathrm{~cm}$. During the acquisitions, we used time-resolved reflectance curves with about 1.5 $\times 10^{6}$ counts (acquisition time: $1 \mathrm{~s}$ for each wavelength). The results, shown in Figs. 4 and 5, refer to only one injection-detection line at, respectively, the two different wavelengths of 690 and $829 \mathrm{~nm}$. The points represent the average over ten repeated measurements on each phantom, the bars on the respective standard deviations.

In Figs. 4(a) and 4(d), the measured absorption (reduced scattering) versus the conventional true absorption (reduced scattering) coefficients are respectively plotted. The solid line in Fig. 4(a) represents the best fit of the first four points obtained averaging over the different phantoms. The solid line in Fig. 4(d) was obtained with the same principle but fitting on the last three points. We chose these fitting points since the diffusion theory works properly in the range of low absorption and high reduced scattering values. The experimental points, for both the coefficients, are well aligned along this line and not much dispersed around it, suggesting a good linearity of the instrument.

In Figs. 4(b) and 4(c), the measured absorption (reduced scattering) versus the true reduced scattering (absorption) coefficients are respectively plotted. The solid lines represent the best fit of the second, third, and fourth points for the same reasons explained before [in Fig. 4(c), it is not considered the first point since the zero point is not well calculated with this theoretical model]. Where the solid lines are not horizontal, there is a coupling between the optical parameters. It happens for high value of the two coefficients, as expected from the validity of the diffusion model. ${ }^{27}$ This behavior does not cause us problems; in fact, typical values for the two optical coefficients of the biological media are about $\mu_{a}=0.14 \mathrm{~cm}^{-1}$ and $\mu_{\mathrm{s}}^{\prime}=10 \mathrm{~cm}^{-1}$ (phantom B3), and in this range we observe no coupling between the optical coefficients and good linearity.

Similar results were found for the data at $829 \mathrm{~nm}$, as shown in Fig. 5. According to the absorption and scattering spectra of the phantoms, the points are shifted toward lower absolute values, even if they preserve the characteristics of linearity showed for the data at $690 \mathrm{~nm}$.

\section{Noise}

Noise test allows to estimate the number of counts of the reflectance curve required to have limited variability of the measurement results due to random effects. In Fig. 6, the coefficient of variation (CV) for the absorption and reduced scattering coefficient at $690 \mathrm{~nm}$ [Fig. 6(a)] and $829 \mathrm{~nm}$ 

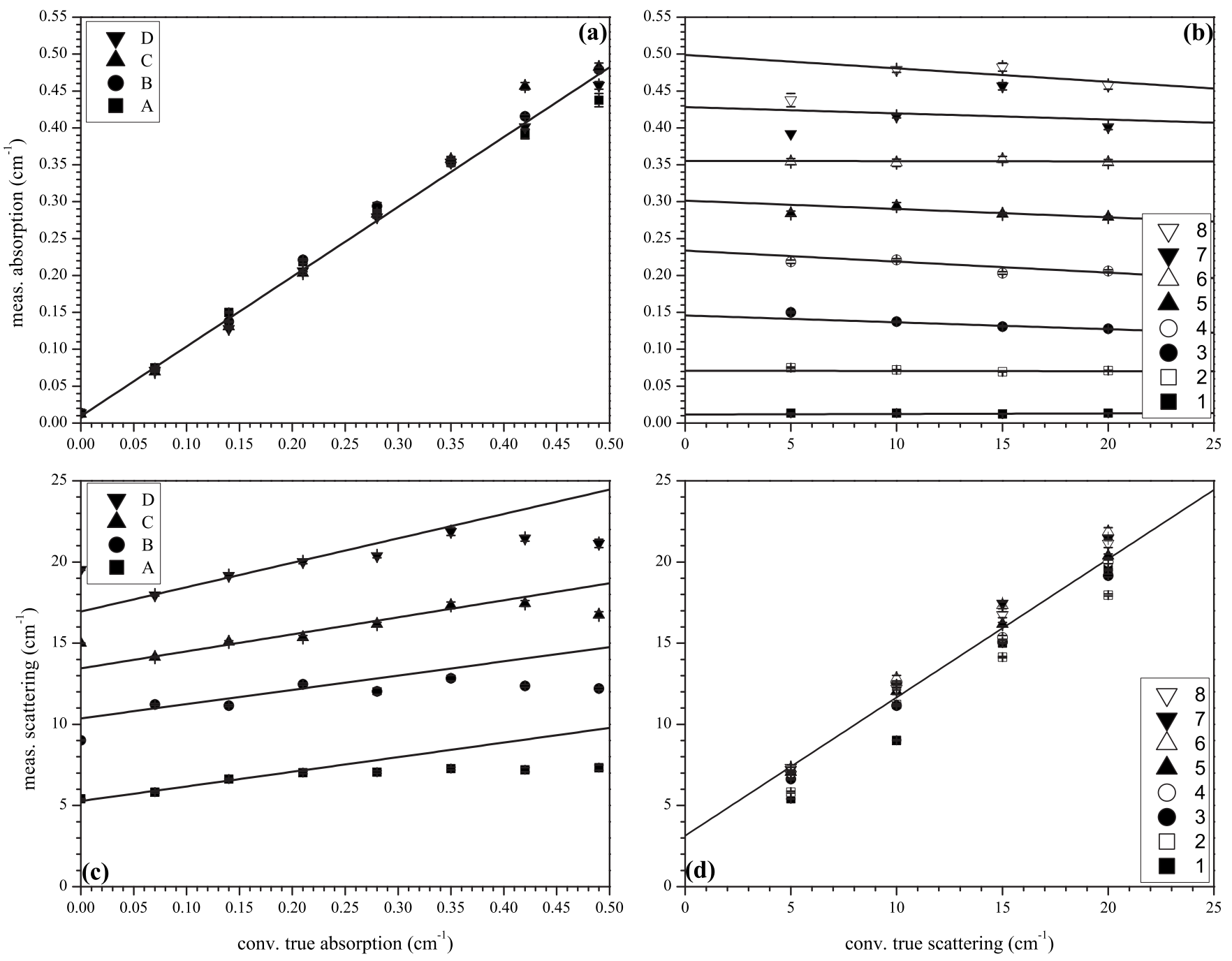

FIG. 4. Linearity for the absorption and reduced scattering coefficients at $690 \mathrm{~nm}$.

[Fig. 6(b)] is shown. We need more than $5 \times 10^{5}\left(7 \times 10^{5}\right)$ counts for the scattering at $690 \mathrm{~nm}$ (absorption at 690 and $829 \mathrm{~nm}$, scattering at $829 \mathrm{~nm}$ ) to have a value of the CV lower than $1 \%$. This is a typical CV value to perform measurements with acceptable precision. Normally, during an in vivo experiment, a collection time of about $200 \mathrm{~ms}$ is sufficient to acquire a curve of about $5 \times 10^{5}$ photons. The solid line is a linear fit of the $\mathrm{CV}$ as function of photons counts $\mathrm{N}$, which has a trend very close to the one indicated by the photon noise statistics. ${ }^{28}$ Indeed, we found a power law dependence: $\mathrm{CV} \div \mathrm{N}^{\mathrm{n}}$, where coefficient $\mathrm{n}=-0.54(\mathrm{n}=-0.54)$ for absorption at $690 \mathrm{~nm}(829 \mathrm{~nm})$ and $\mathrm{n}=-0.57(\mathrm{n}=$ $-0.49)$ for reduced scattering at $690 \mathrm{~nm}(829 \mathrm{~nm})$. We note that the last points, relative to the largest counts, refer to a small number of curves to represent a realistic statistics.

\section{Stability}

The measurements were performed in a reflectance geometry, on phantom B3, with an interfiber distance of $2 \mathrm{~cm}$ and an acquisition time of $2 \mathrm{~s}$ ( $1 \mathrm{~s}$ for each wavelength), for a total of 300 min. In Figs. 7(a) and 7(b), the results of the stability test are expressed as percentage variations with respect to the average value, over the last $30 \mathrm{~min}$, of the ab- sorption coefficient, respectively, at 690 and $829 \mathrm{~nm}$ for one injection-detection line. To reach a stability level of $\pm 3 \%$ $( \pm 1 \%)$ of the average value, the system takes about $24 \mathrm{~min}$ (78 min) being this the warm-up time of laser heads.

\section{Reproducibility}

We performed the same measurements in the same experimental conditions (reflectance geometry, $2 \mathrm{~cm}$ interfiber distance, $1 \mathrm{~s}$ acquisition time, phantom B3) in three different days to evaluate the reproducibility. We calculated the displacement of the measured optical parameters obtained at each day with respect to the value found averaging the values over the days. We found for one injection-detection line displacement of about $8 \%$ (7\%) for the absorption (reduced scattering) coefficient at $690 \mathrm{~nm}$; at $829 \mathrm{~nm}$, we obtained $6 \%$ for both the optical coefficients.

\section{B. Preliminary in vivo measurements}

We performed preliminary tests to check the performances of our instrument during in vivo measurements aiming at monitoring the hemodynamic changes during muscle cuff occlusion and brain activation. Taking the assumption that deoxyhemoglobin and oxyhemoglobin $\left(\mathrm{HHb}\right.$ and $\mathrm{O}_{2} \mathrm{Hb}$, 

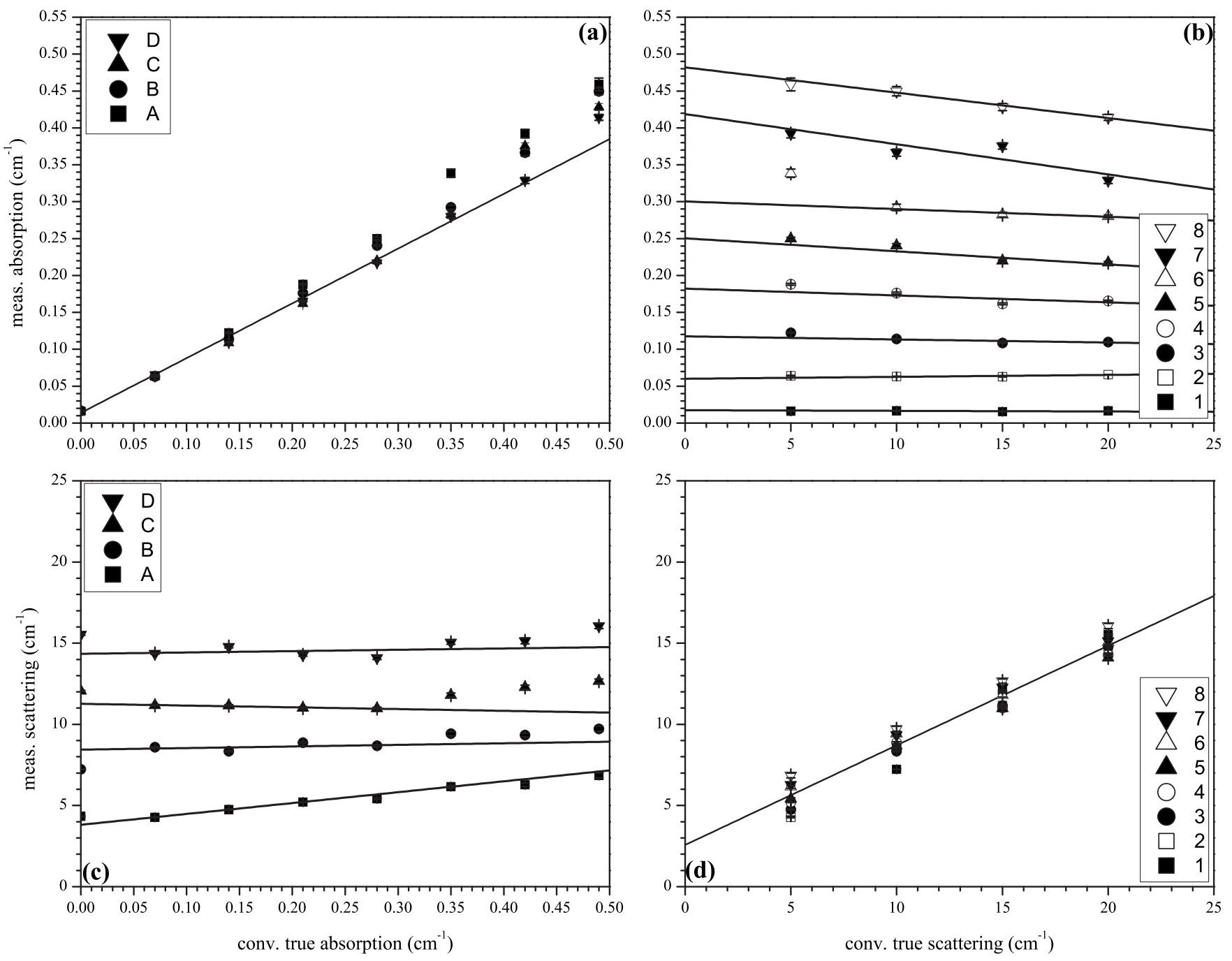

FIG. 5. Linearity for the absorption and reduced scattering coefficients at $829 \mathrm{~nm}$.

respectively) are the main chromophores contributing to absorption, their concentrations are easily derived by using the knowledge of the extinction coefficient. ${ }^{29}$ To enhance the contribution from deep layers and to remove possible disturbances caused by superficial adipose layers, a correction method based on the use of late time windows (17502500 ps) was also applied. ${ }^{30}$

\section{Cuff occlusion on the arm muscle}

A standard arterial cuff occlusion of the arm muscle on a male adult volunteer was performed. The measurement protocol scheduled an arterial occlusion (180 mm Hg, left arm) consisting of $1 \mathrm{~min}$ of baseline, 2 min of task, and $3 \mathrm{~min}$ of recovery, for a total of 6 registration minutes. The measurements were performed in a reflectance geometry, with a sample acquisition rate of $400 \mathrm{~ms}$ (200 ms for each wavelength) and an interfiber distance of $2 \mathrm{~cm}$. We put on the medial aspect of each arm, one injection fiber and one detection bundle by means of a black rubber pad connected to brand fasteners (ONE-WRAP ${ }^{\circledR}$, Velcro Italia Srl, Italy). They guarantee a good adhesion of the fiber to the skin also in the event of movements of the subject. We obtained similar results for all the volunteers.

In Fig. 8(a), the time courses for the variations of $\mathrm{HHb}$ and $\mathrm{O}_{2} \mathrm{Hb}$ on both the arms, calculated as difference with the average values over the first $30 \mathrm{~s}$ of the baseline period, are shown. During the arterial occlusion, the flow of the deoxygenated blood through the veins and the influx of the oxygenated one through the artery are inhibited. In fact, we observe an increase of the $\mathrm{HHb}$ and a decrease of $\mathrm{O}_{2} \mathrm{Hb}$ during the entire occlusion task. On the right arm, relevant variations of the hemodynamic parameters are not present, as expected. When the cuff is released (slow release, in a few seconds, to avoid abrupt changes), we observe a return to the baseline value with the presence of oscillations (i.e., undershoot for $\mathrm{HHb}$ and overshoot for $\mathrm{O}_{2} \mathrm{Hb}$ ). We notice a delay in the response of $\mathrm{HHb}$ compared to $\mathrm{O}_{2} \mathrm{Hb}$, as expected, since the veins are released after the arteries, this effect being enhanced by the slow release of the cuff.

One of the advantages of time-resolved technique is the possibility to extract not only the relative variations, but also the absolute value of the optical parameters. This can be exploited to estimate, as example, the time courses of blood oxygen saturation $\left[\mathrm{SO}_{2}=\mathrm{O}_{2} \mathrm{Hb} /\left(\mathrm{O}_{2} \mathrm{Hb}+\mathrm{HHb}\right)\right]$. In Fig. 8(b), 

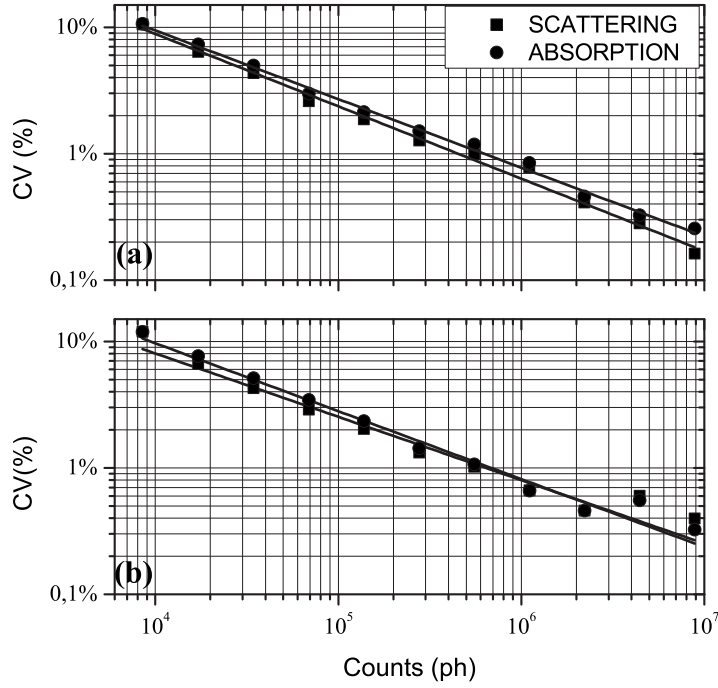

FIG. 6. CV for the absorption and reduced scattering coefficients. The solid lines are the linear fit of CV. (a) CV at $690 \mathrm{~nm}$. (b) CV at $829 \mathrm{~nm}$.

$\mathrm{SO}_{2}$ measured during the arterial occlusion on the left arm is shown. During baseline, $\mathrm{SO}_{2}$ is around $60 \%$, a typical value for human muscle, ${ }^{22,23}$ while a decrease to about $55 \%$ is observed during the arterial occlusion period.

\section{Motor cortex response during hand movements}

We employed our instrument also during an in vivo test on an adult volunteer to study the hemodynamic response of the motor cortex during the movement of the right hand. The injection and detection fibers were arranged on the scalp over the focal point for hand movement (optodes centered across the C3 and C4 positions, as defined by the 10/20 EEG International System) with an interfiber distance of $2 \mathrm{~cm}$. The measurement protocol consisted of $10 \mathrm{~s}$ of baseline, $20 \mathrm{~s}$ of motor task (opening and closing right hand), and $10 \mathrm{~s}$ of recovery. An acquisition time of $1 \mathrm{~s}(0.5 \mathrm{~s}$ for each wavelength) was used. The protocol was repeated 15 times, and the results were averaged.

In Fig. 9, changes in $\mathrm{HHb}(\Delta \mathrm{HHb})$ and $\mathrm{O}_{2} \mathrm{Hb}\left(\Delta \mathrm{O}_{2} \mathrm{Hb}\right)$ during the measurements are plotted. The graph in Fig. 9(a) shows the results for the left hemisphere. Here, it is evident that the increasing of $\mathrm{O}_{2} \mathrm{Hb}$ and the corresponding decreasing of $\mathrm{HHb}$ during the motor task, as expected, by the con-

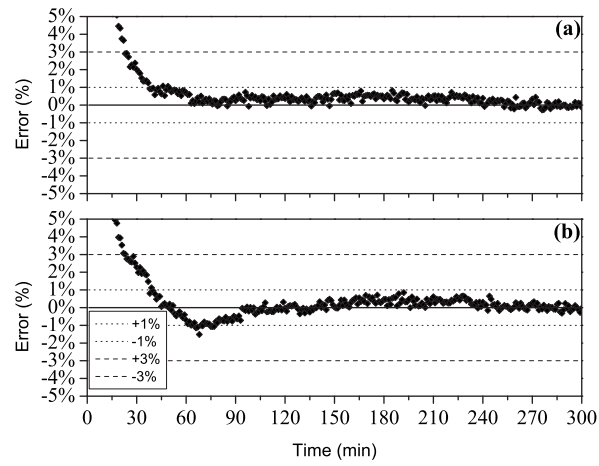

FIG. 7. Stability test: percentage variations with respect of the average values of the absorption coefficients at (a) $690 \mathrm{~nm}$ and (b) $829 \mathrm{~nm}$.
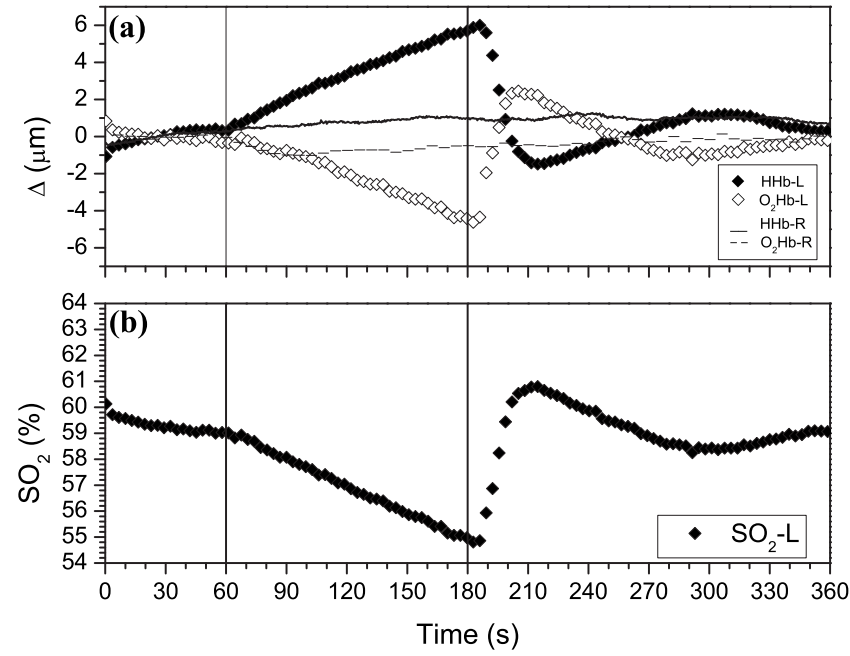

FIG. 8. Arterial occlusion on the left arm. (a) Changes in $\mathrm{HHb}(\Delta \mathrm{HHb})$ and $\mathrm{O}_{2} \mathrm{Hb}\left(\Delta \mathrm{O}_{2} \mathrm{Hb}\right)$ on the left (l) and right (r) arm. (b) Time course of the absolute value of the saturation coefficient $\left(\mathrm{SO}_{2} \%\right)$.

tralateral brain activation. The graph in Fig. 9(b) refers to the right hemisphere, where there are not significant task related changes during the experiment, as expected.

\section{COMPARISON BETWEEN WAVELENGTH SPACE MULTIPLEXING AND TIME MULTIPLEXING APPROACHES}

To quantify the cross-talk between the wavelengths, we have compared the two different approaches to inject light into the tissue in three different configurations: SM, TM in a dual-channel system, and time multiplexing in a system with more channels, where a routing electronics is introduced, time multiplexing with router (TMR). We have done measurements keeping one wavelength $\left(\lambda_{\mathrm{F}}\right)$ at a fixed count rate (four different values: $10^{5}, 5 \times 10^{5}, 10^{6}, \quad 1.5$ $\times 10^{6}$ photons/s) and varying the count rate at the other wavelength $\left(\lambda_{\mathrm{V}}\right)$ (nine values: $\pm 75 \%, \pm 50 \%, \pm 25 \%$, $\pm 10 \%$, and $0 \%$ of the constant value). Figure 10 shows the case for $\lambda_{\mathrm{F}}=690 \mathrm{~nm}$ and $\lambda_{\mathrm{V}}=829 \mathrm{~nm}$. In principle, varying the intensity of $\lambda_{\mathrm{V}}$, we should not observe a variation in the amount of the count rate at $\lambda_{\mathrm{F}}$, and the points in Fig. 10 should be horizontals. This is what happens when the count

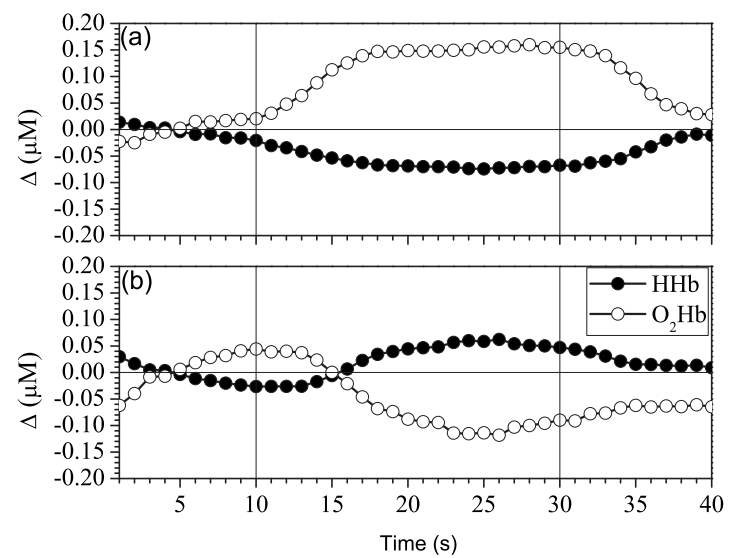

FIG. 9. Changes in $\mathrm{HHb}(\Delta \mathrm{HHb})$ and $\mathrm{O}_{2} \mathrm{Hb}\left(\Delta \mathrm{O}_{2} \mathrm{Hb}\right)$ during right hand movements. (a) Left hemisphere. (b) Right hemisphere. 

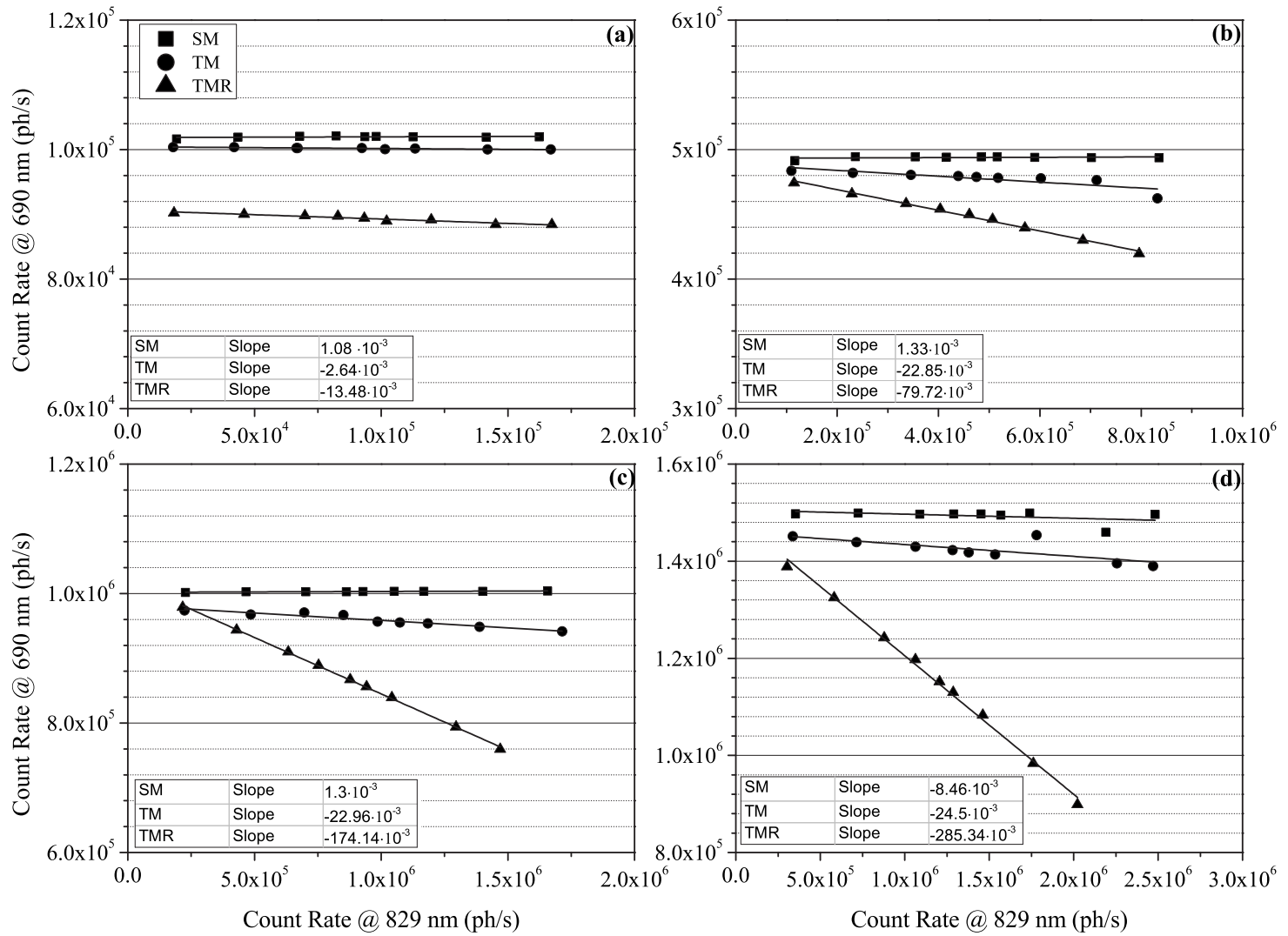

FIG. 10. Trend of the count rate at $690 \mathrm{~nm}$ when it is kept fixed at different values and the count rate at $829 \mathrm{~nm}$ is varied. Fixed values: (a) $10^{5}$ photons/s, (b) $5 \times 10^{5}$ photons $/ \mathrm{s}$, (c) $10^{6}$ photons $/ \mathrm{s}$, and (d) $1.5 \times 10^{6}$ photons $/ \mathrm{s}$. The results are shown for three different configurations: SM, TM, and TMR. The solid lines are the linear fit.

rates are low $\left(\lambda_{\mathrm{F}}\right.$ at $10^{5}$ counts/s) [Fig. 10(a)]. By increasing the value of the counts at $\lambda_{\mathrm{F}}$ [Figs. 10(b)-10(d)], the SM configuration shows the absence of cross-talk between the wavelengths, as expected, since the two curves relative to the two different wavelengths are acquired in two different temporal windows (see linear fit and solid lines). To quantify the cross-talk, a linear regression of the points for each configuration has been performed. The slope parameter (dimensionless) represents the relative change in the acquired photons count rate at $\lambda_{\mathrm{F}}$ with respect to the acquired photon count rate at $\lambda_{\mathrm{V}}$. The slope of the linear fit increases in the TMR configuration already from the second value [Fig. 10(b)]. This behavior is not present in the TM configuration, which has a trend similar to the SM one. This suggests that a routing electronics introduces a saturation threshold, which limits the photons count rate in each temporal window. Therefore, when the number of channels is large, a tradeoff between the number of channels (quality of the imaging) and the count rate (quality of the signal-to-noise ratio) is required.

Then, we have evaluated the effect of the cross-talk on the estimation of the absolute values of the optical parameters. In Fig. 11, we plotted the dependence of the scattering coefficients at $829 \mathrm{~nm}$ at different values of $\lambda_{\mathrm{V}}$. When the count rate at $\lambda_{\mathrm{F}}$ is low, the slopes of the linear fit (solid line) are small-in fact, line are almost horizontals-for all the three different configurations, as shown in Figs. 11(a) and 11(c). When the count rate at $\lambda_{\mathrm{F}}$ is $1.5 \times 10^{6} \mathrm{photons} / \mathrm{s}$, the slopes become higher for the TM configuration and much higher for the TMR configuration. This shows that keeping a high count rate at $\lambda_{\mathrm{F}}$ and varying it at $\lambda_{\mathrm{V}}$, an error in the determination of the absolute value of the scattering coefficient is introduced.

The main reason for this behavior is the partial overlap of the DTOFs (and of possible reflections in the optical system) at the two different wavelengths in the TM and TMR configurations. This could be avoided by reducing the frequency repetition rate. However, not all laser sources can operate at different repetition rates. Furthermore, reducing the repetition rate will not eliminate the cross-talk due to the acquisition of more DTOFs in a single TCSPC window. This behavior is not present in the SM configuration, which exploits the entire TCSPC window for one DTOF at one wavelength at a time. Similar results were obtained for the scattering coefficients at $690 \mathrm{~nm}$ and for the absorption coefficients at both wavelengths.

\section{CONCLUSION}

We have developed and characterized a compact, dualwavelength, and dual-channel system for time-resolved diffuse NIR spectroscopy. It is based on the wavelength space multiplexing approach that allows to avoid cross-talk affecting system based on the wavelength time multiplexing approach and to increase signal-to-noise ratio. The instrument was also validated during preliminary in vivo tests. 

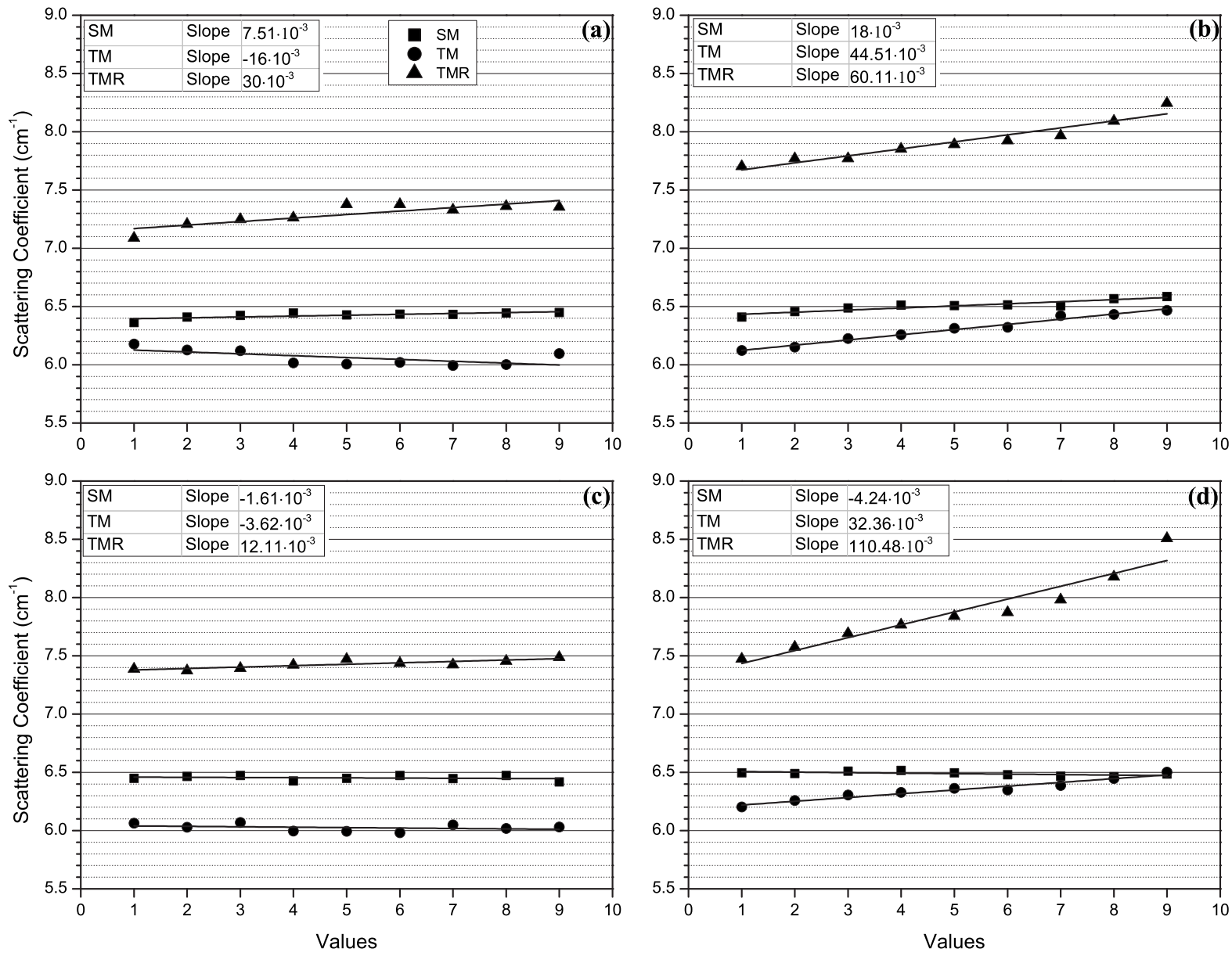

FIG. 11. Scattering coefficients at $829 \mathrm{~nm}$. First row: $\lambda_{\mathrm{F}}=690 \mathrm{~nm}$ and $\lambda_{\mathrm{V}}=829 \mathrm{~nm}$; second row: $\lambda_{\mathrm{F}}=829 \mathrm{~nm}$ and $\lambda_{\mathrm{V}}=690 \mathrm{~nm}$. First column: fixed value of $10^{5}$ photons/s; second column: fixed value of $1.5 \times 10^{6}$ photons $/ \mathrm{s}$. The solid lines are the linear fit.

\section{ACKNOWLEDGMENTS}

This research was partially funded from the European Community's Seventh Framework Programme under the nEUROPt Project (Grant No. FP7- HEALTH-2007-201076).

${ }^{1}$ F. F. Jobsis, Science 198, 1264 (1977).

${ }^{2}$ E. M. C. Hillman, J. Biomed. Opt. 12, 051402 (2007)

${ }^{3}$ T. Durduran, R. Choe, W. B. Baker, and A. G. Yodh, Rep. Prog. Phys. 73, 076701 (2010).

${ }^{4}$ H. Obrig and A. Villringer, J. Cereb. Blood Flow Metab. 23, 1 (2003).

${ }^{5}$ S. Lloyd-Fox, A. Blasi, and C. E. Elwell, Neurosci. Biobehav Rev. 34, 269 (2010).

${ }^{6}$ T. Hamaoka, K. K. McCully, V. Quaresima, K. Yamamoto, and B. Chance, J. Biomed. Opt. 12, 062105 (2007).

${ }^{7}$ H. Liu, D. A. Boas, Y. Zhang, A. G. Yodh, and B. Chance, Phys. Med. Biol. 40, 1983 (1995).

${ }^{8}$ Y. Hoshi, J. Biomed. Opt. 12, 062106 (2007).

${ }^{9}$ S. Fantini and M. A. Franceschini, in Handbook of Optical Biomedical Diagnostics, edited by V. V. Tuchin (SPIE Press, Bellingham, WA, 2002), Chap. 7.

${ }^{10}$ M. Wolf, M. A. Franceschini, L. A. Paunescu, V. Toronov, A. Michalos, U. Wolf, E. Gratton, and S. Fantini, Adv. Exp. Med. Biol. 530, 61 (2003).

${ }^{11}$ T. H. Pham, O. Coquoz, J. B. Fishkin, E. Anderson, and B. Tromberg, Rev. Sci. Instrum. 71, 2500 (2000).

${ }^{12}$ D. Boas, D. Brooks, E. Miller, C. Di Marzio, M. Kilmer, R. Gaudette, and Q. Zhang, IEEE Signal Process. Mag. 18, 57 (2001).

${ }^{13}$ A. Torricelli, D. Contini, A. Pifferi, L. Spinelli, and R. Cubeddu, OptoElectron. Rev. 16, 131 (2008).

${ }^{14}$ M. S. Patterson, B. Chance, and B. C. Wilson, Appl. Opt. 28, 2331 (1989).

${ }^{15}$ J. Steinbrink, H. Wabnitz, H. Obrig, A. Villringer, and H. Rinneberg, Phys. Med. Biol. 46, 879 (2001).
${ }^{16}$ S. Del Bianco, F. Martelli, and G. Zaccanti, Phys. Med. Biol. 47, 4131 (2002).

${ }^{17}$ J. Selb, J. J. Stott, M. A. Franceschini, A. G. Sorensen, and D. A. Boas, J. Biomed. Opt. 10, 011013 (2005).

${ }^{18}$ A. Torricelli, A. Pifferi, L. Spinelli, R. Cubeddu, F. Martelli, S. Del Bianco, and G. Zaccanti, Phys. Rev. Lett. 95, 078101 (2005).

${ }^{19}$ M. Wolf, M. Ferrari, and V. Quaresima, J. Biomed. Opt. 12, 2104 (2007).

${ }^{20}$ M. Kacprzak, A. Liebert, and R. Maniewski, European Conferences on Biomedical Optics, Munich, Germany, 2005.

${ }^{21}$ H. Wabnitz, M. Moeller, A. Liebert, A. Walter, R. Erdmann, O. Raitza, C. Drenckhan, J. P. Dreier, H. Obrig, J. Steinbrink, and R. MacDonald, Proc. SPIE 5859, 58590H (2005).

${ }^{22}$ D. Contini, A. Torricelli, A. Pifferi, L. Spinelli, F. Paglia, and R. Cubeddu, Opt. Express 14, 5418 (2006).

${ }^{23}$ S. Ferrante, D. Contini, L. Spinelli, A. Pedrocchi, A. Torricelli, F. Molteni, and G. Ferrigno, J. Biomed. Opt. 14, 044011 (2009).

${ }^{24}$ W. Becker, Advanced Time-Correlated Single-Photon Counting (SpringerVerlag, Berlin, Germany, 2005).

${ }^{25}$ A. Pifferi, A. Torricelli, A. Bassi, P. Taroni, R. Cubeddu, H. Wabnitz, D. Grosenick, M. Möller, R. Macdonald, J. Swartling, T. Svensson, S. Andersson-Engels, R. L. P. Van Veen, H. J. C. M. Sterenborg, J. M. Tualle, H. L. Nghiem, E. Tinet, S. Avrillier, M. Whelan, and H. Stamm, Appl. Opt., 44, 2104 (2005).

${ }^{26}$ D. Contini, F. Martelli, and G. Zaccanti, Appl. Opt. 36, 4587 (1997).

${ }^{27}$ R. Cubeddu, A. Pifferi, P. Taroni, A. Torricelli, and G. Valentini, Med. Phys. 23, 1625 (1996).

${ }^{28}$ W. Louisell, Quantum Statistical Properties of Radiation (Wiley, New York, 1973).

${ }^{29}$ S. Prahl, Oregon Medical Laser Center; See http://omlc.ogi.edu/spectra.

${ }^{30}$ D. Contini, A. Torricelli, A. Pifferi, L. Spinelli, and R. Cubeddu, Proc. SPIE 6629, 662908 (2007). 